University of Nebraska - Lincoln

DigitalCommons@University of Nebraska - Lincoln

Michigan Bovine Tuberculosis Bibliography and

Database

Wildlife Disease and Zoonotics

2002

\title{
Lesion Development in White-tailed Deer (Odocoileus virginianus) Experimentally Infected with Mycobacterium bovis
}

\author{
M. V. Palmer \\ Bacterial Diseases of Livestock Research Unit, National Animal Disease Center, Agricultural Research \\ Service, US Department of Agriculture,Ames, IA, Mitchell.Palmer@ars.usda.gov \\ W. R. Waters \\ Bacterial Diseases of Livestock Research Unit, National Animal Disease Center, Agricultural Research \\ Service, US Department of Agriculture, Ames, IA \\ D. L. Whipple \\ Bacterial Diseases of Livestock Research Unit, National Animal Disease Center, Agricultural Research \\ Service, US Department of Agriculture, Ames, IA
}

Follow this and additional works at: https://digitalcommons.unl.edu/michbovinetb

Part of the Veterinary Medicine Commons

Palmer, M. V.; Waters, W. R.; and Whipple, D. L., "Lesion Development in White-tailed Deer (Odocoileus virginianus) Experimentally Infected with Mycobacterium bovis" (2002). Michigan Bovine Tuberculosis Bibliography and Database. 77.

https://digitalcommons.unl.edu/michbovinetb/77

This Article is brought to you for free and open access by the Wildlife Disease and Zoonotics at DigitalCommons@University of Nebraska - Lincoln. It has been accepted for inclusion in Michigan Bovine Tuberculosis Bibliography and Database by an authorized administrator of DigitalCommons@University of Nebraska Lincoln. 


\title{
Lesion Development in White-tailed Deer (Odocoileus virginianus) Experimentally Infected with Mycobacterium bovis
}

\author{
M. V. Palmer, W. R. Waters, and D. L. Whipple \\ Bacterial Diseases of Livestock Research Unit, National Animal Disease Center, Agricultural Research Service, \\ US Department of Agriculture, Ames, IA
}

\begin{abstract}
The recent discovery of tuberculosis in free-living white-tailed deer in northeastern Michigan underscores the need for increased understanding of the pathogenesis of tuberculosis in wildlife species. To investigate lesion development in white-tailed deer, 32 deer were experimentally infected by intratonsilar instillation of 300 colony-forming units of Mycobacterium bovis. Three deer each were euthanatized and examined at days 15, 28, 42, and 56 after inoculation, and five deer each were euthanatized and examined at days 89, 180, 262, and 328 after inoculation. Microscopic lesions first were seen in the medial retropharyngeal lymph node and lung 28 and 42 days after inoculation, respectively. Lung lesions were present in 12 (38\%) of 32 deer, involving 23 lung lobes. Left caudal and right middle and caudal lobes were involved in 17 (74\%) of the 23 affected lung lobes. Lesions in the medial retropharyngeal lymph node first appeared as granulomas composed of aggregates of macrophages and Langhans-type giant cells. Some early granulomas contained centrally located neutrophils. As granulomas developed, neutrophils were replaced with a central zone of caseous necrosis that first showed signs of mineralization 42 days after inoculation. Granulomas increased in size as the zone of caseous necrosis expanded. Peripheral fibrosis, first seen at 56 days after inoculation, progressed to only a thin fibrous capsule by 328 days after inoculation. By the termination of the study, the central necrotic core of the granuloma contained abundant liquefied necrotic material and grossly resembled an abscess. Although tuberculous lesions in white-tailed deer follow a developmental pattern similar to that in cattle, fibrosis is less pronounced and the advanced lesions may liquefy, a change seldom reported in cattle. An understanding of lesion development will aid in the identification of the spectrum of disease that may be seen in this important wildlife reservoir of tuberculosis.
\end{abstract}

Key words: Deer; granuloma; Mycobacterium bovis; Odocoileus virginianus; tuberculosis.

In 1994, a free-living white-tailed deer (Odocoileus virginianus) in Michigan was diagnosed with tuberculosis caused by Mycobacterium bovis. ${ }^{22}$ Subsequent surveys conducted by the Michigan Department of Natural Resources and Michigan State University Animal Health Diagnostic Laboratory identified an epidemic of M. bovis infection in free-living white-tailed deer in northeastern Michigan. ${ }^{13,22}$ This represents the first known reservoir of $M$. bovis in free-living wildlife in the United States and the first known epidemic of tuberculosis in white-tailed deer in the world. Several factors are thought to have contributed to the establishment and persistence of $M$. bovis in this wildlife reservoir. These factors include the large number of cattle infected with $M$. bovis in Michigan during the late $1950 \mathrm{~s}^{8}$ and a deer population that has steadily grown beyond normal habitat carrying capacity to focal concentrations of 19-23 deer per $\mathrm{km}^{2} .{ }^{22}$ A contributing factor to overpopulation has been long-term winter feeding to prevent deer migration and decrease winter mortality in order to keep deer numbers high for hunting purposes. ${ }^{22}$ Winter feeding not only results in increased numbers of deer, but causes prolonged crowding of deer around feeding sites with increased opportunity for deer to deer contact and enhanced transmission of tuberculosis. DNA analysis of M. bovis isolates from Michigan white-tailed deer has shown that the majority of deer were infected with a common strain of $M$. bovis, suggesting a single source of infection. ${ }^{27}$ The presence of $M$. bovis in wildlife also represents a serious threat to domestic livestock. Eighteen M. bovis-infected cattle herds have been identified in Michigan since the identification of tuberculosis in white-tailed deer. Restriction fragment length polymorphism analysis of $M$. bovis isolates from deer and cattle show that they are identical, suggesting that cattle became infected through contact with tuberculous white-tailed deer. ${ }^{26}$

Little is known concerning the pathogenesis of tuberculosis in white-tailed deer. Development of diagnostic tests, vaccines, or other tools that may be used in disease control programs will require an improved understanding of the development of tuberculosis in white-tailed deer. This study was conducted to exam- 
ine the development and distribution of lesions in white-tailed deer experimentally infected with $M$. bovis.

\section{Materials and Methods}

\section{Animals}

Thirty-two, 9-month-old female and castrated male whitetailed deer were inoculated intratonsilarly with approximately 150 colony-forming units (CFU) of M. bovis strain 1315 into each tonsilar crypt for a total dose of $300 \mathrm{CFU}$, as described previously. ${ }^{14}$ Strain 1315 was originally isolated in 1995 from a white-tailed deer in Michigan. For intratonsilar inoculation, deer were anesthetized by intramuscular injection of a combination of xylazine $(2 \mathrm{mg} / \mathrm{kg}$; Mobay Corporation, Shawnee, KS) and ketamine $(6 \mathrm{mg} / \mathrm{kg}$; Fort Dodge Laboratories, Fort Dodge, IA). After inoculation, the effects of xylazine were reversed by intravenous (IV) injection of tolazoline (4 mg/kg; Lloyd Laboratories, Shanandoah, IA).

Animals were housed in Association for Assessment and Accreditation of Laboratory Animal Care International approved biosecurity level 3 housing, and fed a commercial pelleted feed with free access to water. An outline of all procedures was approved by the National Animal Disease Center Institutional Animal Care and Use Committee before the beginning of the experiment. Animal euthanasia was conducted in a manner consistent with the guidelines of the American Veterinary Medical Association panel on euthanasia. $^{2}$

Before to inoculation and 90 and 180 days thereafter, delayed-type hypersensitivity (DTH) to $M$. bovis was measured by intradermal skin testing by the comparative cervical skin test (CCT), as described previously. ${ }^{14,17}$

\section{Sampling}

Before inoculation and at 30-day intervals thereafter, swabs of the nasal cavities, oral cavity, and rectum were obtained and processed for isolation of M. bovis, as previously described. ${ }^{14}$ Additionally, to examine the degree of environmental contamination that may result from inoculated deer excreting M. bovis, at 30-day intervals, samples of feed, water, and feces from the floor of each pen were collected and processed as described for the isolation of M. bovis. ${ }^{17}$ Three deer each were euthanatized and examined 15, 28, 42, and 56 days after inoculation. Five deer each were euthanatized and examined 89, 180, 262, and 328 days after inoculation. Animals were anesthetized as above and euthanatized by IV sodium pentobarbital. At necropsy, the following tissues or fluids were collected and processed for isolation of $M$. bovis as described; ${ }^{14}$ palatine tonsil; pharyngeal tonsil; mandibular, parotid, medial retropharyngeal, superficial cervical, tracheobronchial, mediastinal, hepatic, mesenteric, iliac, prefemoral, and popliteal lymph nodes; lung; liver; spleen; kidney; brain; urine; feces; and swabs of the tonsilar crypts and tracheal lumen. Each lung lobe was examined separately and cross-sectioned at $0.5-$ to $1.0-\mathrm{cm}$ intervals. Tissues collected for microscopic analysis were fixed by immersion in $10 \%$ neutral buffered formalin and included all tissues collected for bacteriologic examination as well as adrenal gland, myocardium, abomasum, and rumen. For microscopic examination, formalin-fixed tissues

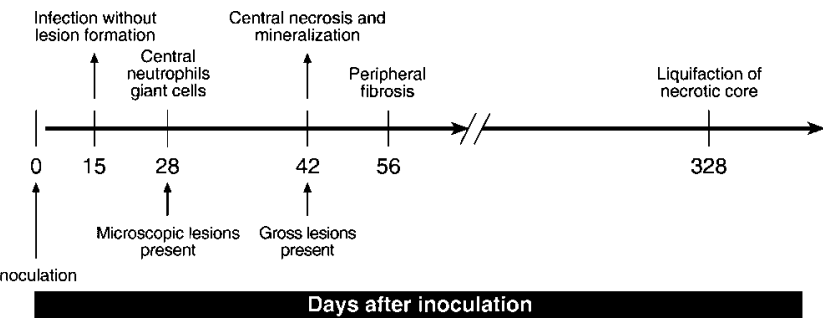

Fig. 1. Schematic representation of the chronology of morphologic changes in the medial retropharyngeal lymph nodes of white-tailed deer inoculated intratonsilarly with 300 $\mathrm{CFU}$ of $M$. bovis and examined at various times after inoculation.

were processed by routine paraffin-embedment techniques, cut in 5- $\mu \mathrm{m}$ sections, and stained with hematoxylin and eosin (HE). Adjacent sections were cut from samples containing lesions suggestive of tuberculosis and stained by the Ziehl-Neelsen technique for identification of acid-fast bacteria. $^{23}$

\section{Results}

Delayed-type hypersensitivity to $M$. bovis was not seen in any deer before inoculation. Ninety and 180 days after inoculation, all experimentally inoculated deer exhibited DTH reactions consistent with exposure to $M$. bovis.

Mycobacterium bovis was not isolated from any sample of feed, water, or feces from the floor of the pens at any time during the experiment. Likewise, $M$. bovis was not isolated from urine, feces, or any swab of the oral cavity, nasal cavity, rectum, or tracheal lumen at any time. However, M. bovis was recovered from a swab of the tonsilar crypt of one deer that was euthanatized at 28 days after inoculation. M. bovis was not isolated from any sample of brain; kidney; spleen; liver; or iliac, prefemoral, popliteal, or superficial cervical lymph nodes.

Mycobacterium bovis was recovered from the palatine tonsil and medial retropharyngeal lymph node at 15 days, the earliest time point examined after inoculation; however, microscopic and gross lesions were not seen until 28 and 42 days, respectively, after inoculation (Fig. 1). Pulmonary lesions were first noted 42 days after inoculation (Table 1). Lesions in the lungs consisted of small, often solitary, 3- to 10-mm pleural and subpleural, white to gray foci, containing dry, sometimes gritty contents (Fig. 2). Although lesions were present in lungs of deer from 42 days after inoculation to the termination of the experiment, the number of pulmonary lesions or their severity did not increase as the experiment progressed. Twelve (38\%) of 32 deer had lung lesions involving 23 separate lung lobes. The left caudal and right middle and caudal lung lobes represented 17 (74\%) of 23 of the affected lobes 


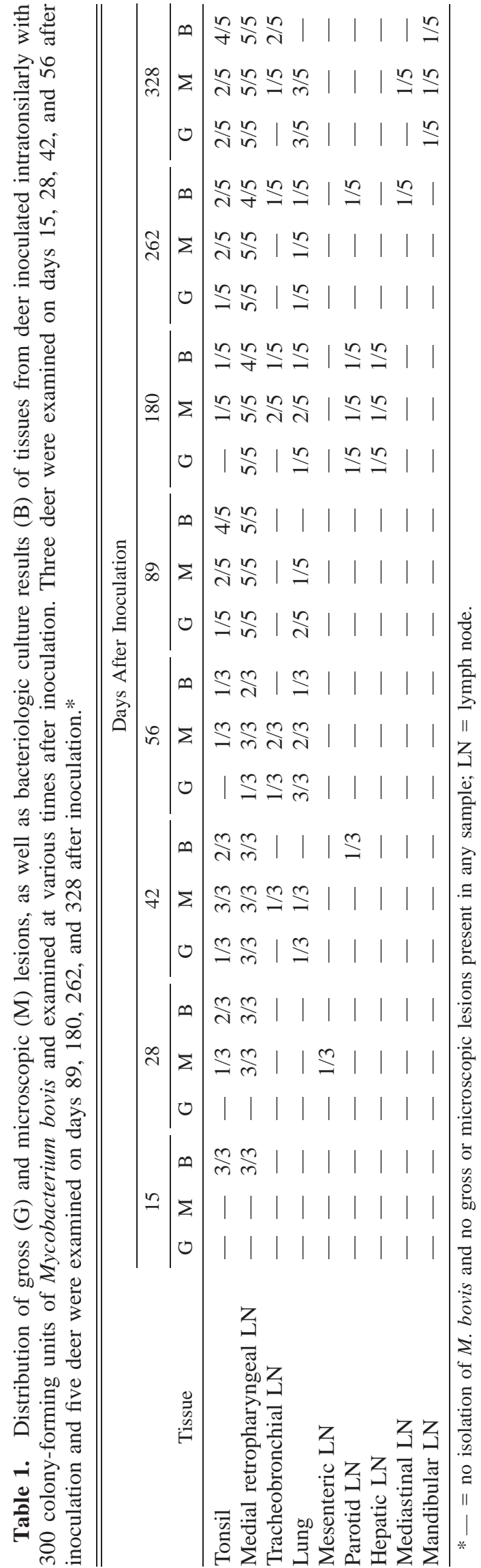

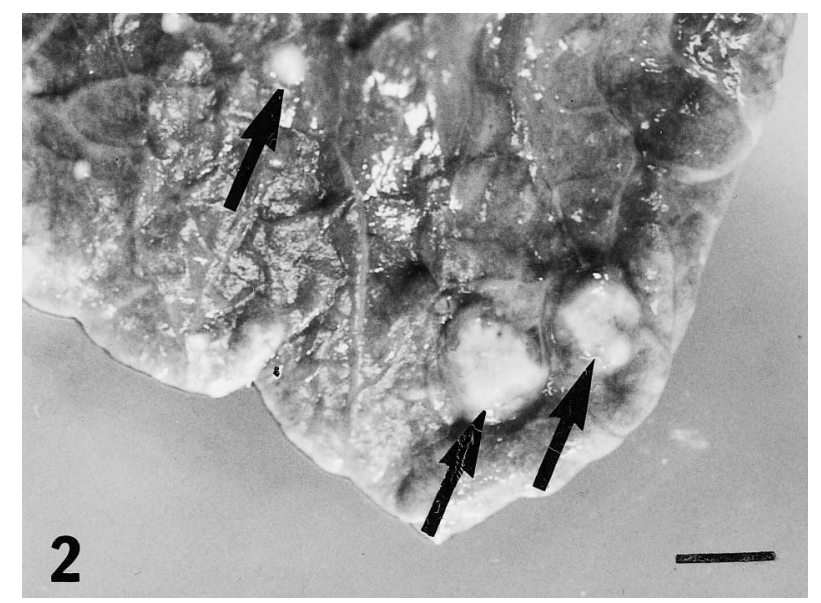

Fig. 2. Lung; white-tailed deer inoculated intratonsilarly with $300 \mathrm{CFU}$ of M. bovis and examined 262 days later. Multifocal, small granulomas on pulmonary surface (arrows). Bar $=12 \mathrm{~mm}$.

(Fig. 3). To examine lesion development over time, medial retropharyngeal lymph nodes were carefully examined at all time points.

Twenty-eight days after inoculation, medial retropharyngeal lymph nodes were of normal size, but firm consistency. Microscopically, granulomas were composed of aggregates of macrophages, Langhans-type multinucleated giant cells, and few lymphocytes (Fig. 4). Some of these early granulomas contained a small, centrally located focus of neutrophils (Fig. 5). Moderate to large numbers of acid-fast bacilli were present within the central neutrophilic infiltrate. No mineralization or evidence of collagen deposition associated with the granuloma was seen.

By 42 days after inoculation, bilateral lymphadenomegaly of the medial retropharyngeal lymph nodes was present. Multifocal, 2- to 5-mm, pale, firm lesions were present within the lymph node cortex. Microscopically, lesions were composed of variably sized, coalescent granulomas composed of aggregates of macrophages, Langhans-type multinucleated giant cells, and low numbers of lymphocytes and neutrophils. A central area of necrosis containing fine granular staining visualized by the Von Kossa method and consistent with early mineralization was present within coalescent granulomas. Evidence of collagen deposition was not seen. Acid-fast bacteria were present in low numbers and seen only within the necrotic core.

Fifty-six through 89 days after inoculation, medial retropharyngeal lymph nodes were bilaterally enlarged and contained multifocal, sometimes coalescent cortical granulomas. Microscopically, lesions consisted of large areas of necrosis surrounded by variable thick bands of cellular infiltrate containing macrophages, Langhans-type multinucleated giant cells, and lympho- 


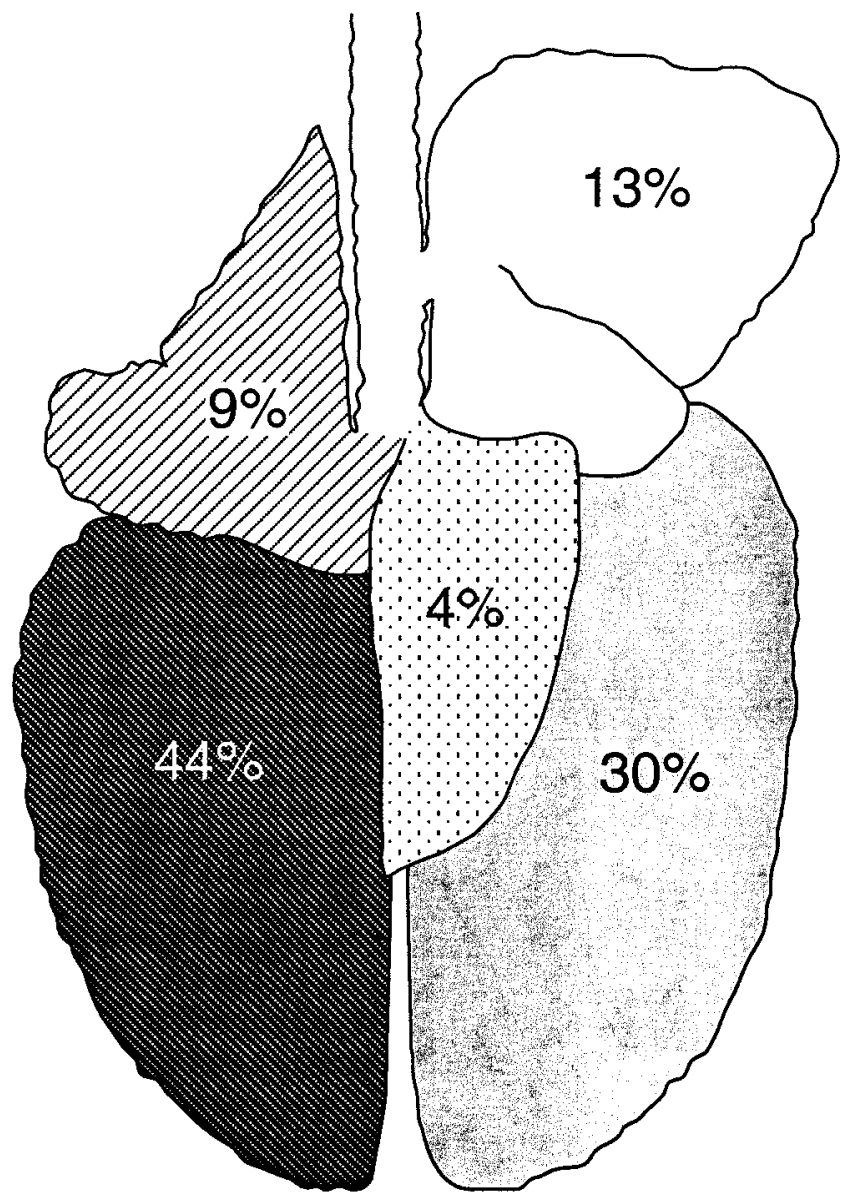

\section{$\square$ right cranial lobe right middle and cranial lobes $\square 7$ left cranial lobe left caudal lobe accessory lobe}

Fig. 3. Schematic of deer lung demonstrating the distribution of lung lesions in white-tailed deer intratonsilarly inoculated with $300 \mathrm{CFU}$ of $M$. bovis and examined at various times after inoculation. Numbers represent the percentage of deer with lesions in a respective lung lobe.

cytes. Von Kossa staining revealed central granular mineralization of necrotic debris. Low to moderate numbers of acid-fast bacilli were present within the mineralized area. Thin bands of collagen, visualized by Masson's trichrome staining, surrounded the granuloma, separating it from unaffected lymph node.

Medial retropharyngeal lymph nodes at 180 days after inoculation, grossly resembled those seen at the previous time point. Lesions were also microscopically similar, with the exception of more expansive regions of necrosis containing partially mineralized debris and moderate numbers of acid-fast bacilli. Necrotic cores were surrounded by variably thick bands of macrophages, Langhans-type multinucleated giant cells, and lymphocytes interspersed in a stroma of loosely arranged collagenous connective tissue.

At 262 days after inoculation, medial retropharyngeal lymph node lesions were similar to those seen at 180 days after inoculation; however, more extensive mineralization of the necrotic debris within the centers of granulomas was present.

Medial retropharyngeal lymph nodes were grossly similar at 328 days after inoculation to those seen at the previous time points; however, on sectioning, many lesions contained central liquefied, purulent material. Microscopically, such lesions were composed of coalescent, expansive, partially mineralized, necrotic material surrounded by a thin rim of macrophages, Langhans-type multinucleated giant cells, and lymphocytes in a loosely arranged fibrous stroma. Moderate numbers of neutrophils were present at the interface between the necrotic core and granulomatous infiltrate. Variable numbers of acid-fast bacteria were present within the mineralized necrotic material.

Lesions in the lung followed the same developmental pattern as did lesions in the medial retropharyngeal lymph nodes with some exceptions. Microscopic lesions were first seen at 42 days after inoculation and mineralization of the necrotic core and evidence of mild peripheral fibrosis were seen on days 56 and 89, respectively, after inoculation. This represented a shift of one time point later than the appearance of such changes in the medial retropharyngeal lymph node. Additionally, although lung lesions progressed to coalescent caseonecrotic granulomas with central mineralization and mild peripheral fibrosis, no evidence of liquefaction of the necrotic core was seen in lung lesions at any time.

\section{Discussion}

Examination of lesion development in the medial retropharyngeal lymph node in the present study is appropriate for two reasons. First, although the tonsil has no afferent lymphatics, the efferent lymphatics drain primarily to the medial retropharyngeal lymph nodes..$^{21}$ Therefore, after intratonsilar inoculation, medial retropharyngeal lymph nodes should be one of the earliest sites of lesion development. Second, the medial retropharyngeal lymph node is often the most commonly affected tissue in naturally infected whitetailed deer. ${ }^{13,15,22}$

The first appearance of microscopic lesions 28 days after intratonsilar inoculation is analogous to that seen in cattle inoculated in similar fashion. ${ }^{16}$ In contrast, cattle inoculated intranasally develop lesions as early 


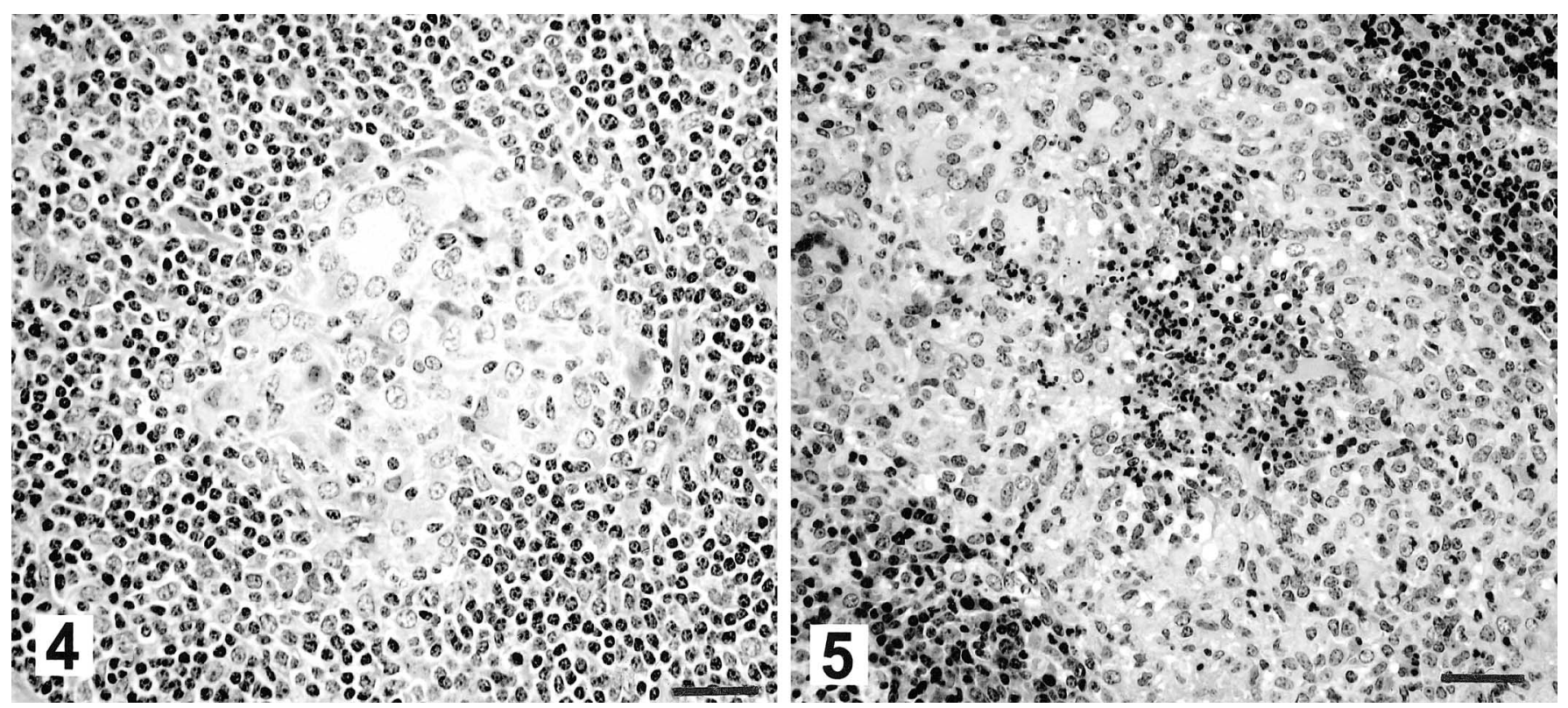

Fig. 4. Medial retropharyngeal lymph node; white-tailed deer inoculated intratonsilarly with $300 \mathrm{CFU}$ of $M$. bovis; 28 days after inoculation. Early granuloma is composed of macrophages and Langhans-type multinucleated giant cells. HE. Bar $=55 \mu \mathrm{m}$.

Fig. 5. Medial retropharyngeal lymph node; white-tailed deer inoculated intratonsilarly with $300 \mathrm{CFU}$ of $M$. bovis; 28 days after inoculation. Centrally located neutrophils are surrounded by macrophages and Langhans-type multinucleated giant cells. HE. Bar $=88 \mu \mathrm{m}$.

as 7 days after inoculation. ${ }^{5}$ This difference may be due to host species differences or differences in route of inoculation, inoculum strain, or inoculum dosage. The cellular constituents of lesions seen in deer in the present study are similar to those seen in cattle. ${ }^{5,16}$ However, in contrast to cattle, in deer, only mild fibrosis surrounding granulomas was present even at 328 days after inoculation. Prominent collagen deposition has been noted in lesions from cattle as early as 56 days after intratonsilar inoculation. ${ }^{16}$

In the present study, intratonsilar inoculation of 300 CFU of $M$. bovis resulted in infection or lesion development in 32 (100\%) of 32 inoculated deer. Similar inoculation of red deer (Cervus elaphus) with dosages of $M$. bovis ranging from 8 to $8,000 \mathrm{CFU}$ resulted in infection of $87 \%$ of inoculated deer. ${ }^{10}$ Much higher dosages, $1.5 \times 10^{5}$ to $2 \times 10^{7} \mathrm{CFU}$, have been used to experimentally infect cattle..$^{5,16}$ Under the conditions outlined in the current study, white-tailed deer seem to be highly susceptible to infection with $M$. bovis.

Twelve $(38 \%)$ of 32 deer in the present study developed lung lesions consistent with tuberculosis. Lung lesions were first seen 42 days after inoculation. In cattle inoculated intranasally, lung lesions are seen as early as 7 days after inoculation. ${ }^{5}$ In cattle examined at an abattoir, lung lesions were reported in $7-17 \%$ of tuberculous cattle. 7,9,25 However, careful examination of the lungs has revealed lung lesions in as many as
$73-89 \%$ of cattle with confirmed tuberculosis. ${ }^{11,24}$ The predilection for lesion development in caudal lung lobes, as seen in the present study, has been reported in tuberculous cattle, where $90 \%$ of pulmonary lesions are found in the diaphragmatic lobes, approximately $50 \%$ of which are in the distal one third. ${ }^{11,12,24}$ The reason for such a predilection is unclear. The delayed appearance of mineralization of the necrotic core and peripheral fibrosis of lung lesions as compared to lesions in the medial retropharyngeal lymph node suggest that lymph node lesions predated the lung lesions. Lung lesions may have developed as sputum containing $M$. bovis was inhaled. Hematogenous spread of $M$. bovis to the lung was less likely because this would result in more numerous lung lesions disseminated widely throughout the lung.

Although the cellular response to $M$. bovis is considered to be of a granulomatous nature, in the present study, the earliest lesions were composed of both macrophages and neutrophils. Later, lesions seen at 328 days after inoculation also contained moderate numbers of neutrophils at the interface between the necrotic core and the granulomatous infiltrate. Neutrophils have been noted in early lesions $(<21$ days after inoculation) of experimentally infected cattle ${ }^{5}$ and bacillus Calmette-Guérir-induced granulomas in experimentally infected mice. ${ }^{18}$ Neutrophils are capable of killing both virulent and avirulent Mycobacterium tu- 
berculosis in vitro ${ }^{4}$ and have been shown to play a protective role in mouse models of tuberculosis. ${ }^{3,19}$

In vitro research on giant cell formation has found that giant cells initially formed are of the foreign body type and those formed later are of the Langhans type. ${ }^{1}$ Giant cells seem to arise from fusion of macrophages and take the form of either foreign body or Langhans type, based on microtubule-mediated reorganization. ${ }^{1}$ Giant cells of the foreign body type were uncommon in the present study, with those seen even in the earliest recognizable granulomas being of the Langhans type. This difference may be due to species differences or differences in the inciting agent used in previous reports.

Necrosis was first seen at 42 days after inoculation. Necrosis seen within granulomas often is coincident with the development of DTH by the host. ${ }^{1}$ In the present study, DTH to $M$. bovis purified protein derivative, as measured by the CCT, was not present before experimental inoculation but was evident in all deer by 90 days after inoculation. However, no assay to measure DTH was done before 90 days after inoculation; therefore, the relationship of DTH development and necrosis within granulomas cannot be elucidated.

The general morphologic changes during early lesion development are similar to those described in intranasally inoculated cattle. ${ }^{5}$ In the present study, by the termination of the experiment, affected lymph nodes were markedly enlarged, often containing abundant purulent exudate surrounded by a granulomatous infiltrate and a light fibrous capsule. Grossly, many of these lesions resembled abscesses. The abscesslike nature of tuberculous granulomas has been identified in naturally infected white-tailed deer and other members of the family Cervidae. ${ }^{15,20}$ Less frequent reports of abscesslike lesions in tuberculous cattle are available. ${ }^{25}$ The conversion of a solid or semisolid caseous lesion to one of a more liquid nature is similar to the liquefaction of tuberculous pulmonary lesions described in humans and experimentally infected rabbits and due to the action of various proteinases and nucleases. ${ }^{6}$ The presence of liquefied lesions is thought to be important in the dissemination of disease within the host as well as to other susceptible hosts.

Previously, white-tailed deer experimentally infected with the same strain of $M$. bovis used in the current study were shown to excrete $M$. bovis in oral and nasal secretions, urine, and feces. ${ }^{14,17}$ In the present study, such excretion was not seen. This discrepancy likely is due to the relative low dose of inoculum (300 CFU) used compared to higher doses $\left(2 \times 10^{3}\right.$ to $2 \times 10^{8}$ CFU) used previously. ${ }^{14,17}$ With lower doses, longer than 328 days may be required for lung lesions to expand and enter airways that can then result in $M$. bovis in tracheal lumina, nasal secretions, or saliva.
The description of the spectrum of lesions that develop in tuberculous white-tailed deer will aid in lesion identification in this important wildlife reservoir of tuberculosis.

\section{Acknowledgements}

We thank Rebecca Lyon, Jody Mentele, Theresa Rahner, and Lori Dethloff for technical assistance, and Katie Lies, Wayne Varland, Doug Ewing, Norm Lyon, and Terry Krausman for animal care. Product names are necessary to report factually on available data; however, the US Department of Agriculture (USDA) neither guarantees nor warrants the standard of the product, and the use of the name by the USDA implies no approval of the product to the exclusion of others that may also be suitable.

\section{References}

1 Adams DO: The granulomatous inflammatory response. Am J Pathol 84:164-191, 1976

2 Anonymous: 1993 report of the AVMA panel on euthanasia. J Am Vet Med Assoc 202:230-249, 1993

3 Appleberg R, Castro AG, Gomes S, Pedrosa J, Silva MT: Susceptibility of beige mice to Mycobacterium avium: role of neutrophils. Infect Immun 63:3381-3387, 1995

4 Brown AE, Holzer TJ, Andersen BR: Capacity of human neutrophils to kill Mycobacterium tuberculosis. J Infect Dis 156:985-989, 1987

5 Cassidy JP, Bryson DG, Pollock JM, Evans RT, Forster $\mathrm{R}$, Neill SD: Early lesion formation in cattle experimentally infected with Mycobacterium bovis. J Comp Pathol 119:27-44, 1998

6 Converse PJ, Dannenberg AM, Estep JE, Sugisaki K, Abe Y, Schofield BH, Pitt LM: Cavitary tuberculosis produced in rabbits by aerosolized virulent tubercle bacilli. Infect Immun 64:4776-4787, 1996

7 Corner LA, Melville L, McCubbin K: Efficiency of inspection procedures for the detection of tuberculosis lesions in cattle. Aust Vet J 67:389-392, 1990

8 Frye GH: Bovine tuberculosis eradication: the program in the United States. In: Mycobacterium bovis Infection in Animals and Humans, ed. Thoen CO and Steele JH, pp. 119-129. Iowa State University Press, Ames, IA, 1995

9 Lepper WD, Pearson CW: The route of infection in tuberculosis in beef cattle. Aust Vet J 49:266-267, 1973

10 Mackintosh C, Waldrup K, Labes R, Buchan G, Griffin F: Intra-tonsil inoculation: an experimental model for tuberculosis in deer. In: Tuberculosis in Wildlife and Domestic Animals, ed. Griffin F and de Lisle G, pp. 121122. Otago University Press, Dunedin, New Zealand, 1995

11 McIlroy SG, Neill SD, McCracken RM: Pulmonary lesions and Mycobacterium bovis excretion from the respiratory tract of tuberculin reacting cattle. Vet Rec 118: 718-721, 1986

12 McKay WM: A clinical study of bovine tuberculosis in Banffshire: the pathologic lesions, part 1. Br Vet J 115: 324-329, 1959

13 O’Brien DJ, Fitzgerald SD, Lyon TJ, Butler KL, Fierke 
JS, Clarke KR, Schmitt SM, Cooley TM, Berry DM. Tuberculous lesions in free-ranging white-tailed deer in Michigan. J Wildl Dis 37:608-613, 2001

14 Palmer MV, Whipple DL, Olsen SC: Development of a model of natural infection with Mycobacterium bovis in white-tailed deer. J Wildl Dis 35:450-457, 1999

15 Palmer MV, Whipple DL, Payeur JP, Alt DP, Esch KJ, Bruning-Fann CS, Kaneene JB: Naturally occurring tuberculosis in white-tailed deer. J Am Vet Med Assoc 216:1921-1924, 2000

16 Palmer MV, Whipple DL, Rhyan JC, Bolin CA, Saari DA: Granuloma development in cattle after intratonsilar inoculation with Mycobacterium bovis. Am J Vet Res 60: 310-315, 1999

17 Palmer MV, Whipple DL, Waters RW: Experimental deer to deer transmission of Mycobacterium bovis. Am J Vet Res 62:692-696, 2001

18 Papadimitriou JM, Spector WG: The ultrastructure of high- and low-turnover inflammatory granulomata. J Pathol 106:37-43, 1972

19 Pedrosa J, Saunders BM, Appelberg R, Orme IM, Silva MT, Cooper AM: Neutrophils play a protective nonphagocytic role in systemic Mycobacterium tuberculosis infection in mice. Infect Immun 68:577-583, 2000

20 Rhyan JC, Saari DA, Williams ES, Miller MW, Davis AJ, Wilson AJ: Gross and microscopic lesions of naturally occurring tuberculosis in a captive herd of wapiti
(Cervus elaphus nelsoni) in Colorado. J Vet Diagn Invest 4:428-433, 1992

21 Saar LL, Getty R. Ruminant lymphatic system. In: The Anatomy of the Domestic Animals, ed. Getty R, 5th ed., p. 1027. WB Saunders, Philadelphia, PA, 1975

22 Schmitt SM, Fitzgerald SD, Cooley TM, Bruning-Fann CS, Sullivan L, Berry D, Carlson T, Minnis RB, Payeur JB, Sikarskie J: Bovine tuberculosis in free-ranging white-tailed deer from Michigan. J Wildl Dis 33:749758, 1997

23 Sheehan DC, Hrapchak BB. Microorganisms. In: Theory and Practice of Histotechnology, 2nd ed., pp. 233-251. Mosby, St. Louis, MO, 1980

24 Stamp JT: Bovine pulmonary tuberculosis. J Comp Pathol 58:9-23, 1948

25 Whipple DL, Bolin CA, Miller JM: Distribution of lesions in cattle infected with Mycobacterium bovis. J Vet Diagn Invest 8:351-354, 1996

26 Whipple DL, Jarnagin JL, Payeur JB: DNA fingerprinting of Mycobacterium bovis isolates from animals in northeast Michigan. Proc Int Symp World Assoc Vet Lab Diagn, p. 83, 1999

27 Whipple DL, Meyer RM, Berry DF, Jarnigan JL, Payeur JB: Molecular epidemiology of tuberculosis in wild white-tailed deer in Michigan and elephants. In: Proceedings United States Animal Health Association, pp. 543-546. Pat Campbell and Associates and Spectrum Press, Richmond, VA, 1997 